\title{
Recent Advances in the Diagnosis of Schistosoma Infection: the Detection of Parasite DNA
}

\begin{abstract}
Ana Rabello/ ${ }^{+}$, Luís André Pontes, Emmanuel Dias-Neto*
Laboratório de Pesquisas Clínicas, Centro de Pesquisas René Rachou-Fiocruz, Av. Augusto de Lima 1715, 30190-002

Belo Horizonte, MG, Brasil *Instituto de Psiquiatria, Faculdade de Medicina, Universidade de São Paulo, São Paulo, SP, Brasil

As Schistosoma sp. control programs are chiefly based on treatment of infected population, adequate case finding has a crucial role. The available diagnostic methods are far from ideal, since the search for eggs in stools and the detection of circulating antigens lack sensitivity in low prevalence and post-treatment situations and antibody detection lacks specificity. In most endemic foci, repeated treatment of infected people leaves a number of non-diagnosed and consequently non-treated persons, enough to maintain a persistent residue of 5 to $10 \%$ prevalence. In an attempt to surpass these diagnostic limitations we have developed a polymerase chain reaction (PCR) for the detection of Schistosoma sp. in feces that, in a first population study, has shown to be more sensitive than three-repeated stool Kato-Katz examination. The PCR may constitute a valuable tool for the diagnosis of the Schistosoma sp. infection in special situations, when high sensitivity and specificity are required and infrastructure is available.
\end{abstract}

Key words: schistosomiais - diagnosis - polymerase chain reaction

Notwithstanding the great number of tests applicable to the schistosomiasis diagnosis, few have resisted to scrutiny of effectiveness, reproducibility, cross reactivity and predictive values. The microscopic demonstration of the parasite's eggs in feces or urine remains the more widespread tool, mainly due to its low operational cost and its feasibility in situations of precarious laboratorial structure. A significant increase in the sensitivity of the parasitological examination may be reached by the exam of a larger number of samples, indicated especially after treatment and in low prevalence settings where expected number of eggs is usually minimal.

Up to the moment antibody detection using crude antigens offers low specificity and consequent low positive predictive value making these methods unsuitable for determination of prevalence and evaluation of control measures.

Detecting eggs or adult worm antigens in urine and sera of Schistosoma sp. infected individuals differentiates past and current infections. The specificity close to $100 \%$ is the main advantage that the detection of circulating antigens offers over the search for antibodies, but the disadvantages include low sensitivity in light infections, high cost, difficult approach and dependence on monoclonal antibodies.

Recently, our group has developed a novel method for the detection of S. mansoni in human samples (Pontes

Financial support: Papes-Fiocruz, CNPq

${ }^{+}$Correspondent author. Fax: +55-31-3295.3115 E-mail: ana@cpqrr.fiocruz.br

Received 18 June 2002

Accepted 15 August 2002 et al. 2002), based on the amplification of a highly repeated DNA sequence. Using a modification of the ROSE method (Steiner et al. 1995), a simple and inexpensive DNA extraction technique and a rapid two step polymerase chain reaction (PCR) it was possible to amplify S. mansoni DNA in human fecal and serum samples. Primers were designed to amplify the $121 \mathrm{bp}$ tandem repeat DNA sequence of S. mansoni described by Hamburger et al. (1991).

The high sensitivity of the approach enabled the detection of the parasite DNA in fecal samples and was confirmed using artificial mixtures of eggs and feces: four positive fecal samples were prepared starting from a sample containing $216 \mathrm{eggs} / \mathrm{g}$ (determined by Kato-Katz stool examination) and mixed to negative feces on ten fold dilutions resulting on samples with estimated concentrations of approximately $21.6,2.16$ and $0.216 \mathrm{eggs} / \mathrm{g}$ of feces. DNA was still detected in the sample containing $2.16 \mathrm{eggs} / \mathrm{g}$ of feces, which makes this technique ten times more sensitive than the Kato-Katz examination. A detection limit of $1 \mathrm{fg}$ of Schistosoma sp. DNA was determined when pure DNA was used as PCR template.

The amplification reaction showed to be specific without cross-reaction with DNA from four related helminthic parasites: Ascaris lumbricoides, Ancylostoma duodenales, Taenia solium, Trichiuris trichiuria.

On a first step evaluation of the efficacy of PCR for a community-based diagnosis (manuscript in preparation), 194 inhabitants from Comercinho, State of Minas Gerais, Brazil, provided three stool samples. Prevalence of infection was $25.3 \%, 29.4 \%$ and $30.9 \%$ examining one, two and three fecal samples by the Kato-Katz method, respectively and $38.1 \%$ examining one fecal sample by the PCR. No eggs were found in the feces of 16 individuals with a positive PCR. On the other hand, two patients with eggs in their stools were PCR negative. 


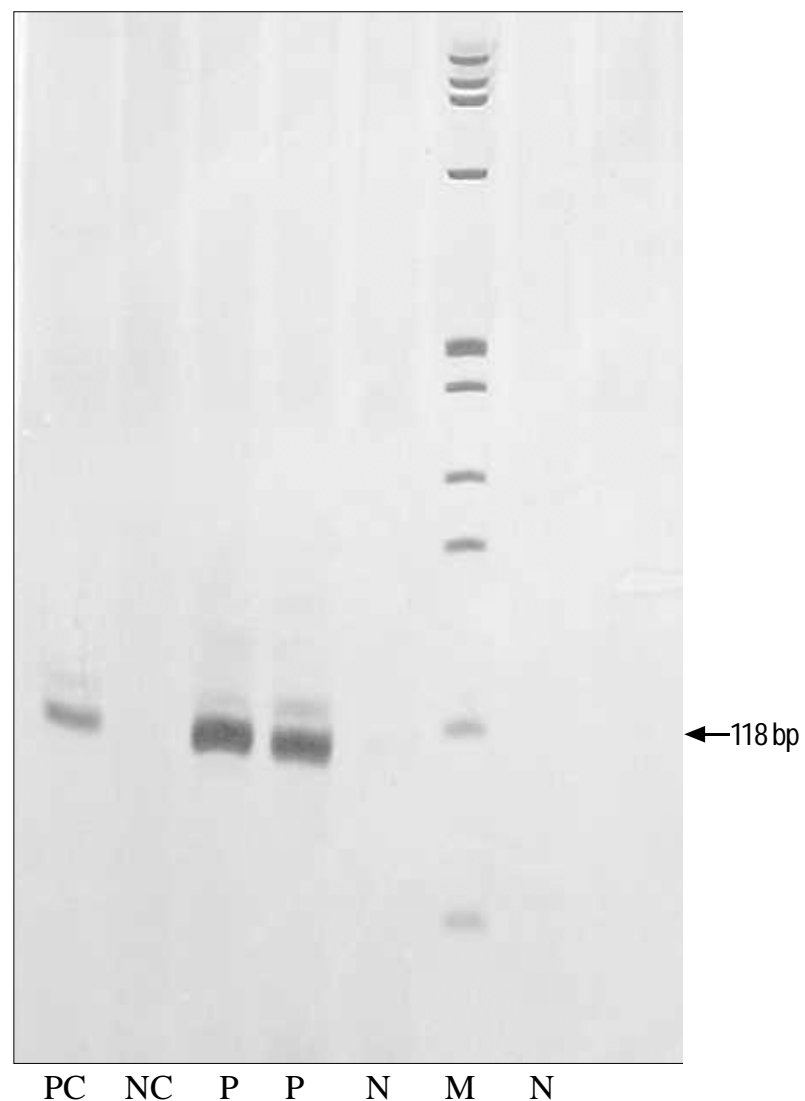

Schistosoma mansoni polymerase chain reaction detection in feces. Lanes - M: molecular weight marker ( $\phi \mathrm{x}-174)$; PC: positive control (S. mansoni egg DNA); NC: negative control; P: positive fecal samples; N: negative fecal samples.

Studies are already underway to ascertain the efficiency of the Schistosoma DNA detection in feces after specific treatment in a population-based study and a kinetic evaluation of the DNA during infection and after treatment in a mouse model.

The DNA amplification assay developed in the two above-mentioned studies may constitute an unprec- edented alternative to the available diagnostic techniques for the detection of $S$. mansoni infection. It demands a more sophisticated laboratory and more complex operational effort, when compared with the Kato-Katz parasitological examination, which surpass both molecular and serological techniques in terms of low costs and ease of operation. Nevertheless, our results indicate that the PCR is more sensitive than the Kato-Katz technique and probably than the circulating antigen detection, which presents a similar sensitivity with the stool examination in situations of low worm burden. The PCR may particularly constitute a tool for the diagnosis of the Schistosoma sp. infection in special situations, when high sensitivity and specificity are required and infrastructure is available (Figure).

This technique is a Fiocruz pending patent. However, it may be used in non-commercial applications and other group's evaluation. There is no need for specific collaboration with the authors as primers and other reagents can be easily purchased. We will be glad to advise on technical details.

In the year 2002, the Centro de Pesquisas René RachouFiocruz celebrates the 30th year of the publication of the Kato-Katz method (Katz et al. 1972), which made possible its use for the diagnosis of schistosomiasis worldwide. We humbly offer the development of this new diagnostic tool as homage to those researchers.

\section{REFERENCES}

Hamburguer J, Turetski T, Kapeller I, Deresiewicz R 1991. Highly repeated short DNA sequences in the genome of Schistosoma mansoni recognized by a species- specific probe. Mol Biochem Parasitol 44: 73-80.

Katz N, Chaves A, Pellegrino J 1972. A simple device for quantitative stool thick smear technique in Schistosomiasis mansoni. Rev Inst Med Trop São Paulo 14: 337-340.

Pontes LA, Dias-Neto E, Rabello A 2002. PCR detection of Schistosoma mansoni DNA in human fecal and serum samples. Am J Trop Med 66: 157-162.

Steiner JJ, Poklemba CJ, Fjellstrom RG, Elliott LF 1995. A rapid one-tube genomic DNA extraction process for PCR and RAPD analyses. $N$ Acid Res 23: 2569-2570. 